\title{
Consilium-Service
}

\section{Kompetente Antworten aus erster Hand}

- Wenn ein Arzt an die Grenzen seines Wissens stößt, kann er die „consilium“-Plattform der Firma InfectoPharm zurate ziehen. Der seit über 25 Jahren etablierte Ärzteservice vermittelt Stellungnahmen von Experten und recherchiert wissenschaftlich-medizinische Daten zu Fragen aus den Bereichen Pädiatrie, Dermatologie, HNOHeilkunde und nun auch Geriatrie.

Viele können mittels der großen Literaturdatenbank, die über 25.000 wissenschaftliche Publikationen und mehr als 8.000 Expertenstellungnahmen umfasst, direkt beantwortet werden. Alle anderen bearbeiten mehr als 300 Experten.

Aus diesen Expertenstellungnahmen werden spezielle Antworten ausgewählt und regelmäßig in Fragen-\&-Antworten-Heften zusammengestellt. Außerdem werden exklusive Themenhefte $\mathrm{zu}$ wichtigen Indikationen in den oben genannten Fachgebieten entwickelt, die besondere Relevanz für den Praxisalltag geachtet. Alle „consilium“-Hefte enthalten ein zertifiziertes CME-Modul, mit dem jeweils 2 Fortbildungspunkte erworben werden können.

$\mathrm{Zu}$ den Servicematerialien gehören auch leicht verständliche Patientenratgeber. Das Servicematerial für Fachkreise (Anmeldung als Angehöriger der Heilberufe erforderlich) bietet für Ärzte, Apotheker und Hebammen maßgeschneiderte Informationen, z. B. zur individuellen Dosierung der Präparate sowie zu Therapierichtlinien für häufige Krankheitsbilder. Alle Servicematerialien und „consilium“-Hefte stehen als Download zur Verfügung.

Red.

- Nach Informationen von InfectoPharm

\section{Kurz notiert}

Tetravalenter Grippeimpfstoff von GSK ab sofort verfügbar $\rightarrow$ Der tetravalente Grippeimpfstoff, Influsplit Tetra ${ }^{\circledR}$ 2016/2017, steht seit Mitte August für die kommende saisonale Grippeimpfung zur Verfügung. In der vergangenen Grippesaison 2015/2016 gab es einen ausgeprägten B-Stamm-Mismatch beim Einsatz trivalenter Grippeimpfstoffe: Über 50\% der Erkrankungsfälle waren auf die im trivalenten Impfstoff fehlende Influenza-B-Linie zurück zu führen. Tetravalente Impfstoffe mit vier statt drei Impfstämmen richten sich entsprechend den aktuellen Empfehlungen der Weltgesundheitsorganisation WHO auch gegen die zweite B-Linie. Um für die kalte Jahreszeit optimal geschützt zu sein, sollte bereits in der infektarmen Spätsommerzeit mit der Impfung begonnen werden. In Deutschland werden die Kosten für Influsplit Tetra ${ }^{\circledR}$ von den gesetzlichen Krankenkassen nur denjenigen Patienten erstattet, bei denen ein schwerer Krankheitsverlauf zu befürchten ist.

- Nach Informationen von GSK

\section{Eisenmangel}

\section{Behandeln, bevor eine Anämie entsteht}

_ In den letzten Jahren ist der „prälatente Eisenmangel“" ohne bereits aufgetretene Anämie ins Blickfeld der Forschung gerückt. Dabei kann es bei normalen Hämoglobin(Hb)-Werten und FerritinWerten unter $50 \mu \mathrm{g} / \mathrm{l} \mathrm{zu}$ therapiebedürftigen Beschwerden kommen. Diese manifestieren sich z. B. in Müdigkeit, Antriebslosigkeit, verminderter Konzentrationsfähigkeit und reduzierter Muskelleistung. Studienergebnisse zeigen, dass von 6.600 Frauen im gebärfähigen Alter ca. $25 \%$ einen Eisenmangel aufweisen, aber nur bei einem geringen Anteil auch eine Anämie diagnostiziert wird [Galan $P$ et al. EJCN. 1998;52:383-8].

Eine Studie mit Eisen(II)-sulfat (Tardyferon $^{\circledR}$ ) mit 198 Frauen im Alter von 18-53 Jahren konnte zeigen, dass eine Eisensubstitution auch bei nicht anämischen Frauen mit unerklärter Fatigue positive Effekte hat [Vaucher P. et al. CMAJ. 2012; doi: 10.1503/cmaj.110950].
Die Frauen erhielten 12 Wochen lang einmal täglich $80 \mathrm{mg}$ Eisen(II)-sulfat oder Placebo. Primärer Endpunkt war eine Besserung der Fatigue, gemessen anhand eines Patienten-Fragebogens (Current and Past Psychological Scale [CAPPS]). Sekundäre Endpunkte waren weitere Scores für Fatigue, Lebensqualität, Depression und Ängstlichkeit sowie diverse Blutparameter.

Nach 12-wöchiger Behandlung zeigte sich eine signifikante Verbesserung der Beschwerden: Der Fatigue-Score nahm in der Verum-Gruppe $(\mathrm{n}=96)$ um 47,7\% ab (vs. 28.8\% unter Placebo). Auch die weiteren Parameter für Fatigue (MAFScale) verbesserten sich unter der Eisentherapie. Bezüglich Lebensqualität, Depression und Ängstlichkeit ergaben sich keine Unterschiede. Bereits nach sechs Wochen konnte eine deutliche Verbesserung der Blutwerte bei den Frauen der Verumgruppe festgestellt

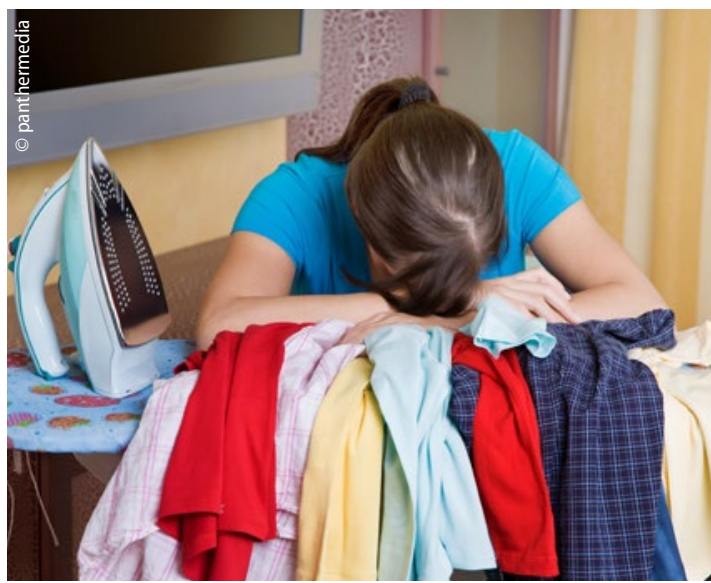

Hat sie einen Eisenmangel?

werden. Das Eisenpräparat war genauso gut verträglich wie Placebo.

Daher empfehlen die Autoren bei Frauen mit normalen Hämoglobinwerten und Ferritinspiegeln unter $50 \mu \mathrm{g} / \mathrm{l}$ eine Eisensubstitution, wenn sich keinerlei sekundäre Auslöser für die $\mathrm{Fa}$ tigue feststellen lassen.

Red.

- Nach Informationen von Pierre Fabre 